\title{
Categories, continua and the growth of psychiatric knowledge
}

\author{
Paul Bebbington
}

Received: 4 December 2014/ Accepted: 14 December 2014/Published online: 25 February 2015

(C) Springer-Verlag Berlin Heidelberg 2015

The four articles commissioned for this issue of Social Psychiatry and Psychiatric Epidemiology are all concerned with the difficulties created for the process of psychiatric classification by the distribution of mental phenomena. I will use the individual papers as the basis of an overall evaluation of the problem of classification in the imperfect world of psychiatry. In particular, what are classifications for, how good can they be, how helpful can they be, and in what ways?

\section{Classification and disease theories}

Because it encapsulates the concept of disease, classification is the central feature of the medical approach towards issues of health. The division of ill-health into categories is based on the belief that this will ultimately enable the rational allotment of treatments. This in turn is because the categories are held to reflect inherent processes (reflected as aetiology and pathology) that might in theory provide targets for specific treatments. Disease classes (syndromes) are constructed when diligent observation identifies groups of people whose ill-health is associated with consistent and distinguishable features, that is, specific symptoms and signs. In this view, disease classes are theoretical constructs that provide a basis for theories of aetiology, pathology, treatment, course and outcome, which can then be tested [1].

Disease classes should always be tentative, and may be revised or abandoned in the light of empirical evidence. When the theories based on them are corroborated, as they

P. Bebbington $(\square)$

Division of Psychiatry, Faculty of Brain Sciences, University College London, Charles Bell House, 67-73 Riding House St., London W1W 7EJ, UK

e-mail: p.bebbington@ucl.ac.uk often are in general medicine, the aetiology or pathology associated with the syndrome may consequently take over as classifiers. It may be, however, as Goldberg argues [2], that psychiatric syndromes are particularly insecure. The strong evidence for underlying dimensions presented by Eaton et al. [3], Carragher et al. [4], and Böhnke and Croudace [5] is a reflection of the central difficulties for classification, which are particularly salient in psychiatry: the boundary and threshold problems, and the artificiality of the distinction between Axis I and Axis II disorders. Nevertheless, it is generally appropriate to reject the theories developed in relation to a given syndrome before proceeding to jettison the syndrome itself as unhelpful.

The scientific utility of psychiatric classes is complicated by the fact that they have social functions, linked to, but separate from, their medical functions of deciding on treatment and care, and identifying cases for research purposes. These other functions are not always benign (they may form the basis of stigma and self-stigmatisation), but they include access to resources in both disorder-driven and problem-driven health systems, the economic justification for health service provision and initiatives, and legal processes both civil and criminal. In particular, drug company licenses are predicated on success in treatment within current classes. Thus the revision of classificatory systems may have non-medical consequences.

\section{Categories and dimensions: the nature of psychiatric symptoms}

Clinical disorders (DSM Axis 1) are almost entirely defined in terms of mental symptoms rather than signs, whereas behavioural disturbance is central to personality disorders (Axis 2). While things can always be classified, it seems 
very likely that classificatory systems in psychiatry will always be unsatisfactory because of the way in which symptoms are defined.

The recognition of a given psychiatric disorder requires that the combination of defining symptoms is sufficient to meet the required criterion. Implicit in this is the existence of combinations of symptoms that fall short of the criterion, and therefore a degree of continuity between case and non-case groups. This is the threshold problem. It is further compounded by the fact that the identification of psychiatric symptoms generally involves the ascription of a cutoff point to what is essentially a continuum. Thus identifying the symptom of depressed mood requires a judgement that the lowering of mood is sufficiently severe, sufficiently persistent, and sufficiently consistent to qualify. Other depressive symptoms are similarly problematic: low self-esteem, loss of confidence, poor concentration, impaired sleep, early-morning wakening, loss of libido, loss of interest and so on. Equivalent judgements must be made about the symptom of anxiety, although, as Goldberg suggests [2], the categorical distinction between phobic and non-phobic anxiety may be relatively secure. Delusions too have dimensional attributes, and grandiose and persecutory delusions in particular shade into ordinary beliefs. Hallucinations and disorders of the experience of thought can be distinguished more categorically from normal experience, although there are still issues of frequency.

Furthermore, some psychiatric symptoms are conceptually linked to the environment. Thus judgements have traditionally been made about whether the depressed mood or anxiety is a normal response, i.e. commensurate with an external provocation. Hence the inordinate tangle over the distinction between depressive disorder and the grief of bereavement.

Finally, the distinction between psychiatric symptoms and personality traits also involves judgements of persistence and consistency, and this makes for difficulties in distinguishing between clinical disorders and personality disorders.

The issue of continuity also affects the identification of recovery and relapse, wherein issues of duration and severity of symptoms again apply [6]. Should recovery be defined as entry into a state where the operational definition of disorder is no longer met? At what point should treatment be discontinued? How should we view symptomatic states that are below threshold in people who have previously met diagnostic criteria? At what point should a recovered person be seen as having relapsed?

\section{Comorbidity}

Goldberg [2, 7] provides a strong critique of our current classifications in terms of the empirical status of comorbidity. As he points out, a good classification has points of rarity between classes. Comorbidity in the individual case will then be truly informative, not the artefact of a spurious separation. However, in psychiatry points of rarity rarely exist: hence the boundary problem. Disorders are recognised by core (defining) features, but ancillary symptoms are the rule. In some cases, these may be used as an exclusionary clause in the definition of the disorder, in other cases they are discounted as incidental. However, symptoms ancillary to one disorder may be defining to another, thus forming the basis of what Goldberg would argue is a non-informative comorbidity [2].

Carragher et al. [4], like Goldberg, regard high rates of comorbidity as an invitation to re-conceive our views of psychiatric disorder. They offer an explanation of comorbidity between common mental disorders, arguing that it is to be accounted for by underlying externalising and internalising dimensions. These are correlated, and in different combinations may result in disturbances equivalent to particular disorders. Eaton and colleagues [3] provide exhaustive evidence of such transdiagnostic factors underpinning the phenomenon of comorbidity, both between individual clinical disorders, and between clinical disorders and personality disorders. These factors may be more stable than the disorders they are associated with, although they too attenuate with time. A bifactor model (i.e. a general factor plus the externalising/internalising dimensions) may be more appropriate, with the general factor capturing the shared variance in the dimensions. However, Böhnke and Croudace [5] sound a note of warning, providing detailed methodological caveats for this sort of research, in particular the possibility of inconsistent results arising from sample selection and the choice of models. It should be noted that the relationship between internalising and externalising dimensions may be non-reflexive: the behaviour picked up in the latter may arise from the mental symptoms that make up the former, whereas the reverse relationship may be much weaker.

Goldberg takes the phenomenon of comorbidity as an argument for rationalising common mental disorders in terms of an overarching anxious depression domain, together with single symptom qualifiers such as obsession or panic. Goldberg's suggestion of a more flexible approach, recognising the pragmatic value of symptom complexes without reifying them as separate disorders, is sensible and appropriate, though it may be resisted by many working in the field.

\section{Topography and mechanism}

The separation of core and ancillary symptoms in the definition of disorder has been an essentially topographical 
exercise, as has the identification of latent variables. Both have been structural rather than developmental: they are generally assessed cross-sectionally in relation to points in time or periods, but they are rarely analysed in terms of the interplay of factors over time.

I would argue that it is much better to look for potential processes. The non-core attributes of syndromes may indicate mechanisms, and these might be used as targets of psychological treatments, as has happened in psychosis [8]. Likewise, if we knew more about the detailed effects of pharmaceutical treatments in the sequence of recovery, we could also use them more rationally.

The creation of topographical syndrome categories may also have practical consequences, leading clinicians to overvalue what is in the syndrome and to undervalue symptoms that lie outside it.

\section{Classification and treatment}

Treatment decisions are essentially categorical: we decide to treat, or we decide not to. The process of diagnosis is partly intended to inform such decisions by specifying (or at least narrowing) treatment options. In fact, it does so only in the broadest terms. This is reflected in the development of needs for care approaches, which are attempts to circumvent the limitations of classification in the determination of treatment and care [9, 10]. Judgements about needs for care are ideographic rather than nomothetic, and become more subtle the more knowledge we have about individual patients and the more interventions we have available. This is difficult to integrate into health systems that are too prescriptive, i.e. if based predominantly on diagnosis. The existence of sub-threshold and transboundary symptoms also implies the possibility of widening treatment targets.

However, dimensional approaches also have their problems. While it is clear that internalisation and externalisation operate, one cannot treat a latent class. Carragher et al. [4] suggest that dimensions may be interpreted in terms of empirically based thresholds, but establishing such thresholds would be extremely difficult.

\section{Classification and epidemiology}

Classification lies at the heart of psychiatric epidemiology. However, the imprecision in our existing classificatory systems has perhaps had less adverse effect than might be feared. This is because cases that just fail to make the cut are likely to resemble cases that do make it. Certainly the current classes have been the basis of huge advances in psychiatric knowledge in the last 15 years (indeed they enabled us to find out about internalising/externalising dimensions). This is in part precisely because they are unsatisfactory: wrangling with our conceptualisations generates knowledge. In my view, epidemiology will benefit from using both classes and dimensions and by triangulating between the two positions.

\section{Tolerance of ambiguity}

Classifications are always imperfect. This does not necessarily mean the attempt to classify should be abandoned, although we should be modest in our aspirations. Karl Popper [11] warned about the dangers of essentialism, the attempt to use definition to catch the Platonic essence of a concept. The incessant urge to revise psychiatric classifications has something of this flavour. Popper's view was that definitions need only to be good enough to work with empirically. Goldberg argues that categories (in ICD) should aim for maximum reliability and validity based on consensus, and this is probably ambition enough.

On this basis I think we should relax about the imperfections of our classificatory systems, use the information about their imperfections to shape how we evaluate the problems of our patients, assess them in detail, and use that information as the basis of the most rational treatment plan. Certainly we should not change classifications too often.

Conflict of interest The author has no conflict of interest.

\section{References}

1. Bebbington PE (2011) John Wing and the perils of nosolatry. Soc Psychiatry Psychiatr Epidemiol 46:443-446

2. Goldberg D (2015) Psychopathology and classification in psychiatry. Soc Psychiatry Psychiatr Epidemiol 50:1-5

3. Eaton NR, Rodriguez-Seijas C, Carragher N, Krueger RF (2015) Trans diagnostic factors of psychopathology and substance use disorders: a review. Soc Psychiatry Psychiatr Epidemiol 50(2):171-182. doi:10.1007/s00127-014-1001-2

4. Carragher N, Krueger RF, Eaton NR, Slade T (2015) Disorders without borders: current and future directions in the meta-structure of mental disorders. Soc Psychiatry Psychiatr Epidemiol. doi:10.1007/s00127-014-1004-z

5. Böhnke JR, Croudace TJ (2015) Factors of psychological distress: clinical value, measurement substance, and methodological artefacts. Soc Psychiatry Psychiatr Epidemiol. doi:10.1007/ s00127-015-1022-5

6. Bebbington PE, Craig T, Garety P, Fowler D, Dunn G, Colbert S, Fornells-Ambrojo M, Kuipers E (2006) Remission and relapse in psychosis: operational definitions based on case-note data. Psychol Med 36:1551-1562

7. Das-Munshi J, Goldberg D, Bebbington P, Bhugra D, Brugha T, Dewey ME, Jenkins R, Stewart R, Prince M (2008) The public health significance of mixed anxiety and depressive disorder. Beyond current classification. Br J Psychiatry 192:171-177 
8. Freeman D, Garety P (2014) Advances in understanding and treating persecutory delusions: a review. Soc Psychiatry Psychiatr Epidemiol 49:1179-1189

9. Brewin CR, Wing JK, Mangen SP, Brugha TS, MacCarthy B, Lesage A (1988) Needs for care among the long-term mentally ill: a report from the Camberwell High Contact Survey. Psychol Med 18:457-468
10. Bebbington PE, Marsden L, Brewin C, Lesage A (1996) Measuring the need for psychiatric treatment in the general population: the Community Version of the MRC needs for care assessment. Psychol Med 26:229-236

11. Popper KR (1963) Conjectures and refutations: the growth of scientific knowledge. Routledge and Keegan Paul, London 